FUB-HEP 10/93

October 23, 2018

\title{
Monte Carlo Study of Topological Defects in the 3D Heisenberg Model*
}

\author{
Christian Holm ${ }^{1}$ and Wolfhard Janke ${ }^{2}$ \\ 1 Institut für Theoretische Physik, Freie Universität Berlin \\ Arnimallee 14, 1000 Berlin 33, Germany \\ 2 Institut für Physik, Johannes Gutenberg-Universität Mainz \\ Staudinger Weg 7, 6500 Mainz 1, Germany
}

\begin{abstract}
We use single-cluster Monte Carlo simulations to study the role of topological defects in the three-dimensional classical Heisenberg model on simple cubic lattices of size up to $80^{3}$. By applying reweighting techniques to time series generated in the vicinity of the approximate infinite volume transition point $K_{c}$, we obtain clear evidence that the temperature derivative of the average defect density $d\langle n\rangle / d T$ behaves qualitatively like the specific heat, i.e., both observables are finite in the infinite volume limit. This is in contrast to results by Lau and Dasgupta [Phys. Rev. B39 (1989) 7212] who extrapolated a divergent behavior of $d\langle n\rangle / d T$ at $K_{c}$ from simulations on lattices of size up to $16^{3}$. We obtain weak evidence that $d\langle n\rangle / d T$ scales with the same critical exponent as the specific heat. As a byproduct of our simulations, we obtain a very accurate estimate for the ratio $\alpha / \nu$ of the specific-heat exponent with the correlation-length exponent from a finite-size scaling analysis of the energy.
\end{abstract}

PACS number(s): 75.10.Hk, 02.70.Lq, 75.40.Mg, 05.70.Fh

*Work supported in part by Deutsche Forschungsgemeinschaft under grant Kl256. 


\section{Introduction}

It is well known that topological defects can play an important role in phase transitions. $^{1,2}$ Extensively studied examples of systems with pointlike defects are the two-dimensional (2D) XY model ${ }^{3}$ and defect models for 2D melting. ${ }^{2,4}$ Recently Lau and Dasgupta (LD) ${ }^{5}$ have used Monte Carlo (MC) simulations to study the role of topological defects in the three-dimensional (3D) classical Heisenberg model, where the defects are also point-like objects with a binding energy that increases linearly with the separation. ${ }^{6}$ Motivated by the importance of vortex points in the 2D XY model, LD tried to set up a similar pictorial description of the phase transition in the 3D Heisenberg model. Analyzing their simulations on simple cubic (sc) lattices of size $V=L^{3}$ with $L=8,12$ and 16 , LD claimed that the temperature derivative of the average defect density, $\langle n\rangle$, diverges at the critical temperature $T_{c}$ like $d\langle n\rangle / d T \sim t^{-\psi}, t=\left|T-T_{c}\right| / T_{c}$, with an exponent $\psi \approx 0.65$. They further speculated that $\psi=1-\beta$, where $\beta \approx 0.36$ is the critical exponent of the magnetization, and then argued that $\langle n\rangle$ should behave like a "disorder" parameter.

At first sight, the existence of such a strong divergence of $d\langle n\rangle / d T$ seems unlikely, because the definition of defects is quasi-local. It is therefore more likely $^{7}$ that $\langle n\rangle$ should qualitatively behave like the energy and $d\langle n\rangle / d T$ like the specific heat, which is a finite quantity for the 3D Heisenberg model.

Using standard finite-size scaling (FSS) arguments we hence expect to see on finite lattices either

$$
d\langle n\rangle / d T=L^{\psi / \nu} f(x)
$$

or, if the second argument holds true,

$$
d\langle n\rangle / d T=\text { const }+L^{\alpha / \nu} g(x),
$$

where $\nu \approx 0.7$ and $\alpha \approx-0.1$ are the correlation length and specific heat exponents, respectively, $x=t L^{1 / \nu}$ is the finite-size scaling variable, and $f(x), g(x)$ are scaling functions. At fixed $x$, Ansatz (1) predicts an approximate linear divergence in $L, d\langle n\rangle / d T \sim L^{\psi / \nu} \approx L$. On the other hand, because $\alpha$ is negative for the 3D Heisenberg model, Ansatz (2) predicts a constant asymptotic value, $d\langle n\rangle / d T \sim$ const. For sufficiently large $L$ a clear distinction between (1) and (2) should hence be observable.

To decide between the two alternatives we have performed MC simulations on large lattices of size up to $L=80$, employing the single-cluster 
update algorithm ${ }^{8}$ and reweighting techniques. ${ }^{9}$ As a result we find unambiguous support for the second alternative, $d\langle n\rangle / d T \sim$ const, as $L \longrightarrow \infty$.

As a byproduct of our simulations we numerically extrapolate $\alpha / \nu$ from a FSS of the energy in close vicinity of the critical coupling $K_{c}$ with a much higher accuracy than obtained in recent high-precision MC studies. ${ }^{10,11}$

\section{The simulation}

The partition function of the Heisenberg model is given by

$$
Z=\prod_{i}\left[\int \frac{d \Omega_{i}}{4 \pi}\right] \exp (-K E) ; \quad E=\sum_{\langle i, j\rangle}\left(1-\vec{s}_{i} \cdot \vec{s}_{j}\right) .
$$

where $K \equiv J / k_{B} T$ is the (reduced) inverse temperature, $\vec{s}_{i}$ are three-dimensional unit vectors at the sites $i$ of a sc lattice, and $\langle i, j\rangle$ denotes nearest-neighbor pairs. Using the single-cluster update algorithm ${ }^{8}$ we have simulated the partition function (3) for lattices of size $V=L^{3}$ with $L=8,12,16,20,24,32,40$, $48,56,64,72,80$ and periodic boundary conditions. Our main emphasis was on the defect density $n=\sum q^{2} n_{|q|}$, where $n_{1}, n_{2}, \ldots$ are defect densities of charge $q= \pm 1, \pm 2, \ldots$. To locate these charges we followed the definition of Berg and Lüscher ${ }^{12}$ according to which the charge $q_{i^{*}}$ at the dual lattice site $i^{*}$ is given by

$$
q_{i^{*}}=\frac{1}{4 \pi} \sum_{i=1}^{12} A_{i}
$$

with

$$
\cos \left(\frac{1}{2} A_{i}\right)=\frac{1+\vec{s}_{1} \cdot \vec{s}_{2}+\vec{s}_{2} \cdot \vec{s}_{3}+\vec{s}_{3} \cdot \vec{s}_{1}}{\sqrt{2\left(1+\vec{s}_{1} \cdot \vec{s}_{2}\right)\left(1+\vec{s}_{2} \cdot \vec{s}_{3}\right)\left(1+\vec{s}_{3} \cdot \vec{s}_{1}\right)}}
$$

and the $\operatorname{sign}$ of $A_{i}$ is determined by $\operatorname{sign} A_{i}=\operatorname{sign}\left(\vec{s}_{1} \cdot\left(\vec{s}_{2} \times \vec{s}_{3}\right)\right)$. For a sc lattice the distinction between $i$ and $i^{*}$ is inessential, since the difference is only a uniform translation along the space diagonal. The sum $\sum_{i=1}^{12}$ in (4) refers to the 12 triangles that can be formed on the faces of the cube enclosing $q_{i^{*}}$. In (5) the spins at the corners of these triangles are numbered in a counter-clockwise sense relative to the outward pointing normal. For the orientation of the diagonals on each face of the cube we used the convention that they run from the lattice point $i$ to $i+\boldsymbol{e}_{i}+\boldsymbol{e}_{j}$, and from $i+\boldsymbol{e}_{i}$ to 
$i+\boldsymbol{e}_{i}+\boldsymbol{e}_{j}+\boldsymbol{e}_{k}$, where $\boldsymbol{e}_{i}, i=1,2,3$ denote unit-vectors in the three coordinate directions. This choice is obviously not unique, but we have checked that other conventions give on the average the same charges within the error bars. From the definition of $q_{i^{*}}$ it is clear, that a trivial upper bound on the magnitude of the lattice topological charge is $q_{i^{*}} \leq 5$. In our runs the highest topological charge observed was three, which occured on the order of $10^{-7}$ per site and measurement, see Table 1. The likelihood of the appearence of the higher charges was probably too small for them to occur during our run times.

All runs were performed close to the approximate infinite volume transition point $K_{c}=0.6930$, as determined in recent MC studies ${ }^{10,11,13}$ of this model. Since we wanted to have reference data we performed our simulation at the same coupling $K_{0}=0.6929$ as in our study of ref., ${ }^{11}$ which is close enough to $K_{c}$ to allow safe reweighting of our data to this value of $K_{c}$. Because the computation of $q_{i^{*}}$ is quite complex and thus time consuming, we have performed many cluster update steps between measurements, adjusted in such a way that the (integrated) autocorrelation time of the charge density measurements is around $\tau_{n} \approx 1-2$. Since it turned out that the (integrated) autocorrelation times $\tau_{n}$ and $\tau_{\chi}$ of the charge density and the magnetic susceptibility are roughly equal, we were able to guess the required measurement interval by extrapolating our previous results for $\tau_{\chi}{ }^{11}$ to larger lattice sizes $L$. The measurement statistics are given in Table 1 . While the statistics is comparable to that of our previous studies, ${ }^{11}$ and much better than that of LD, we note, that our investigated lattices have much larger linear size up to $L=80$ as compared to $L=48$ in our previous work, and compared to the largest size $L=16$ of LD. For each run we recorded the time series of the energy density $e=E / V$, the magnetization density $m=\left|\sum_{i} \vec{s}_{i}\right| / V$, and the charge densities $n_{|q|}$. The resulting averages $\langle e\rangle,\langle n\rangle$, and $\left\langle n_{|q|}\right\rangle$ can be found in Table 1.

To compute the specific heat $C=d\langle e\rangle / d T$, the thermal expansion coefficient $C_{q}=T d\langle n\rangle / d T$, and the topological susceptibility $\chi_{q}=d\langle n\rangle / d \mu$, where $\mu$ is the "field" in a fugacity term $\mu \sum_{i^{*}} q_{i^{*}}^{2}$ which one can imagine adding to the energy in (3), and $n$ is defined as $n=n_{1}+4 n_{2}+9 n_{3}+\ldots$, we used the relations

$$
\begin{aligned}
C & =V K^{2}\left(\left\langle e^{2}\right\rangle-\langle e\rangle^{2}\right)=V K^{2}\langle e ; e\rangle, \\
C_{q} & =V K(\langle e n\rangle-\langle e\rangle\langle n\rangle)=V K\langle e ; n\rangle,
\end{aligned}
$$




$$
\chi_{q}=V\left(\left\langle n^{2}\right\rangle-\langle n\rangle^{2}\right)=V\langle n ; n\rangle .
$$

To obtain results for the various observables $\mathcal{O}$ at $K$ values in an interval around the simulation point $K_{0}=0.6929$, we applied the reweighting method. ${ }^{9}$ Since we recorded the time series this amounts to computing

$$
\left.\langle\mathcal{O}\rangle\right|_{K}=\frac{\left.\left\langle\mathcal{O} e^{-\Delta K E}\right\rangle\right|_{K_{0}}}{\left.\left\langle e^{-\Delta K E}\right\rangle\right|_{K_{0}}},
$$

with $\Delta K=K-K_{0}$. To obtain errors we devided each run into 20 blocks and used standard Jackknife errors. ${ }^{14}$

The results for the quantities in (6) - (8) at $K_{c}=0.6930$ are collected in Table 2. Also given are the eigenvalues $\lambda_{1}, \lambda_{2}$ of the $2 \times 2$ covariance matrix $M$ of $e$ and $n$ with elements $M_{11}=V K^{2}\langle e ; e\rangle, M_{12}=M_{21}=V K\langle e ; n\rangle$, $M_{22}=V\langle n ; n\rangle$.

\section{Results}

Applying (9) we have determined the temperature dependence of the quantities in (6)-(8). For small lattices, $C_{q}$ has its peak location at temperatures larger than $T_{c}$, in contrast to $C$, which peaks at temperature values smaller than $T_{c}$. With increasing lattice size, however, we observe a strong correlation between $C_{q}$ and $C$, that is, both quantities develop a smooth peak at roughly the same temperature $\left(T<T_{c}\right)$, see Fig. 1 . In contrast to $C$ the peak locations of $C_{q}$ scale non-monotonically, with a crossover at $L \approx 20$.

We focussed first on the scaling behavior of $C_{q}$ at our previous estimate of the critical coupling $K_{c}=0.6930$, obtained from the crossings of the Binder parameter $U=1-\left\langle m^{4}\right\rangle / 3\left\langle m^{2}\right\rangle^{2}{ }^{11}$ Our new data for $U$ on the large lattices confirmed the constancy of our previous result of $U^{*}=0.6217(8)$ and hence our estimate for $K_{c}$. We checked first a scaling Ansatz for $C_{q}$ of the form

$$
C_{q}=C_{q}^{\mathrm{reg}}-a_{0} L^{\alpha^{\prime} / \nu},
$$

where $C_{q}^{\text {reg }}$ is a regular background term which is assumed to be independent of lattice size. ${ }^{15}$ Note that this Ansatz covers both scaling hypotheses (1) and (2). The resulting fit shown in Fig. 2(a) yields $\alpha^{\prime} / \nu=-0.401(61)$, $C_{q}^{\text {reg }}=1.50(8)$, and $a_{0}=1.82(6)$, with a quality factor ${ }^{16} Q=0.30$. The good quality of the fit basically rules out the divergence predicted by the Ansatz 
(1) of LD, and strongly favours (2), which predicts a finite asymptotic value for $C_{q}$. Only if one assumes that the FSS behavior sets in at extremely large lattice sizes, one could still attain an assertion of the form (1), but with the consequence of an extremely small exponent $\psi$. We also tried to reproduce the exponent $\psi \approx 0.65$ of $\mathrm{LD}$, by selecting only their lattices sizes, and fitting a straight line to our first 3 data points. But even then we obtain a much smaller value of $\psi / \nu \approx 0.36(3)$, leading to $\psi \approx 0.25(3)$. We think, this discrepancy to the result of LD is partly due to our higher statistics and partly due to the fact that we obtained $C_{q}$ through a thermodynamic derivation, which normally gives better results than the numerical differentiation used by LD.

Because the Ansatz (2), which was based on the assumption that $\langle n\rangle$ should behave like the energy, fits so well, one can ask, if $\alpha^{\prime}$ is equal to the specific-heat exponent $\alpha$. Using our earlier MC result ${ }^{11}$ of $\nu=0.704(6)$, we get a value of $\alpha^{\prime}=-0.282(46)$, which does, on the first glance, not strongly support this conjecture. The best field theoretical estimates are $\nu=0.705(3)$, $\alpha=-0.115(9)$, and $\alpha / \nu=-0.163(12)$ (resummed perturbation series ${ }^{17}$ ), while our earlier MC study ${ }^{11}$ yielded $\nu=0.704(6), \alpha=-0.112(18)$, and $\alpha / \nu=-0.159(24)$. However, the accuracy of the values of $\alpha$ is somewhat misleading, because they were obtained from hyperscaling, $\alpha=2-3 \nu$. The directly measured values have much larger error bars, for example $\alpha / \nu=$ $-0.30(6)^{10}$ and $\alpha / \nu=-0.33(22) .{ }^{11}$

To compare $\alpha^{\prime}$ directly with the measured specific-heat exponent of the present MC simulation, we fitted $C$ to

$$
C=C^{\mathrm{reg}}-b_{0} L^{\alpha / \nu},
$$

with a constant background term $C^{\text {reg }}{ }^{15}$ The resulting fit in Fig. 2(b) yields $\alpha / \nu=-0.225(80), C^{\mathrm{reg}}=4.8(7)$, and $b_{0}=4.1(5)$ with $Q=0.55$, leading to $\alpha=-0.158(59)$. These values are in very good agreement with the hyperscaling prediction, but noteworthy is also the tendency for the values to come out too large.

Of course, a fit of a divergent quantity, like the first derivative $d C_{q} / d T$, for example, is in principle numerically much easier to handle. We tried to do this for $d C_{q} / d T$ and $d C / d T$ at $K_{c}$, and observed the expected divergent scaling behavior, but unfortunately the statistical errors of the third cumulants involved turned out to be much too large to allow for meaningful fits. 
Other estimates for $\alpha$ and $\alpha^{\prime}$ can be obtained by means of fits of $\langle e\rangle$ and $\langle n\rangle$, which again look qualitatively very alike. According to (11), we should have on periodic lattices a scaling behavior of the energy density $\langle e\rangle$ of the form ${ }^{15,18}$

$$
\langle e\rangle=\langle e\rangle^{\mathrm{reg}}-d_{0} L^{(\alpha-1) / \nu} .
$$

and because of (10) the topological charge density $\langle n\rangle$ should then accordingly scale like

$$
\langle n\rangle=\langle n\rangle^{\mathrm{reg}}-c_{0} L^{\left(\alpha^{\prime}-1\right) / \nu} .
$$

Fits of these quantities at $K_{c}=0.6930$, shown in Fig. 3, yield $\left(\alpha^{\prime}-1\right) / \nu=$ $-1.547(15),\langle n\rangle^{\mathrm{reg}}=0.1074(1)$, and $c_{0}=0.42(2)$, with $Q=0.30$, and $(\alpha-$ $1) / \nu=-1.586(19),\langle e\rangle^{\text {reg }}=2.0106(1)$, and $d_{0}=1.68(8)$, with $Q=0.25$. This results in $\alpha^{\prime} / \nu=-0.127(27), \alpha^{\prime}=-0.089(20), \alpha / \nu=-0.166(31)$, and $\alpha=-0.117(23)$. The results for $\alpha$ and $\alpha / \nu$ are in excellent agreement with the hyperscaling prediction, and have not been directly measured before with such a high precision. We attribute this to our large lattice sizes used, but also to the fact that we used fits of $\langle e\rangle$ instead of $C$. The results for $\alpha^{\prime}$ and $\alpha^{\prime} / \nu$ are now lower than those obtained in (10), but now they are almost consistent with the values for $\alpha$ and $\alpha / \nu$. Still it is a little bit puzzling that both estimates for the exponent $\alpha^{\prime} / \nu$ obtained from the fits (10) and (13) do not agree in their respective error range. We attribute this partly to the unknown FSS behavior of the regular background term $\langle n\rangle^{\text {reg }}$, and partly to the fact that the statistical errors of the three parameter fits should be taken with great care.

We further looked at the scaling behavior of $\chi_{q}$, defined in eq.(8). A first look at the plots suggests to try again a scaling Ansatz of the form

$$
\chi_{q}=\chi_{q}^{\mathrm{reg}}-e_{0} L^{\alpha^{\prime \prime} / \nu} .
$$

From a three-parameter fit we obtain $\alpha^{\prime \prime} / \nu=-0.554(57), \chi_{q}^{\text {reg }}=0.67(2)$, and $e_{0}=0.95(6)$ with $Q=0.41$, leading to $\alpha^{\prime \prime}=-0.390(44)$. This time it seems already very unlikely, that $\alpha^{\prime \prime}$ is equal to the specific-heat exponent. However, if one discards the two lowest $L$ values from the fit, one observes a clear trend towards a lower $\alpha^{\prime \prime}$-value, but with the drawback of increased error bars and no improvement in $\chi^{2} /$ dof (per degree of freedom).

We also checked in all other fits if there were corrections to FSS, by discarding successively the data points for $L=8$ and $L=12$. We observed 
in all quantities a trend to the value of $\alpha / \nu$ predicted by hyperscaling, but at the price of much larger error bars. Also the $\chi^{2} /$ dof did not improve. We further checked for confluent corrections, ${ }^{19}$ by including a term of the form $a_{1} L^{-\omega}$, with $\omega=\Delta / \nu$ fixed at the literature value 0.78. ${ }^{17}$ But again the fits were too unstable to give conclusive results.

We also tested if our results depended strongly on the choice of $K_{c}$, by repeating the fits of all quantities at $K_{c} \pm 0.0002$. The resulting parameters were always consistent with the values at $K_{c}$ in the one- $\sigma$ range.

To get a clearer picture we further looked at the scaling behavior of the eigenvalues of the covariance matrix $M$ of $e$ and $n$, defined by equations (6) - (8), which give two uncorrelated observables $\lambda_{1}$ and $\lambda_{2}$. Again we used a scaling Ansatz of the form

$$
\lambda_{i}=\lambda_{i}^{\mathrm{reg}}-a_{i} L^{\alpha_{i} / \nu}
$$

As results we obtain $\alpha_{1} / \nu=-0.273(73), \lambda_{1}^{\text {reg }}=5.1(5)$, and $a_{1}=4.7(2)$, with $Q=0.49$ and $\alpha_{2} / \nu=-1.45(42), \lambda_{2}^{\text {reg }}=0.1307(8)$, and $a_{2}=0.2(2)$, with $Q=0.60$, leading to $\alpha_{1}=-0.192(54)$ and $\alpha_{2}=-1.02(31)$. This suggests $\alpha_{1} \approx \alpha$ and $\alpha_{2} \approx \alpha-1$. The quality of the fits can be inspected in Fig. 4. The existence of an uncorrelated observable which scales with an exponent different from $\alpha$ suggests that there is possibly a new scaling field, and that $C_{q}$ and $\chi_{q}$ see remnants of this scaling field in their FSS behavior. This becomes particularly clear if one remembers that $\lambda_{1}+\lambda_{2}=C+\chi_{q}$. Therefore at least $\chi_{q}$ should see something of the exponent $\alpha_{2} \approx \alpha-1$. Another alternative would be, that $C_{q}$ and $\chi_{q}$ do not scale directly with $\alpha / \nu$ but with some rational multiple of $\alpha / \nu$. As long as there is no satisfactory theory of the scaling of topological quantities, however, one cannot decide between these alternatives.

\section{Concluding remarks}

We have shown that in the three-dimensional classical Heisenberg model the topological defect density $\langle n\rangle$ and its temperature derivative $C_{q}$ behave qualitatively like the energy $\langle e\rangle$ and its temperature derivative $C$. Especially, we can reject the conjecture of $\mathrm{LD}$ that $C_{q}$ diverges with a new critical exponent $\psi$. 
Rather, our simulations indicate that $C_{q}$ behaves also quantitatively like the specific heat, i.e. scales like (2). We obtain weak evidence that asymptotically for large $L$ the scaling of $C_{q}$ is governed by the specific-heat critical exponent $\alpha$. Still, it cannot be ruled out that the scaling of $C_{q}$ involves also a new exponent belonging to a new scaling field. For the topological susceptibility $\chi_{q}$ we find that it also remains finite, and that it can be fitted with an Ansatz of the form (2) as well, but that its scaling exponent must be some multiple of $\alpha$, be the representative of a new scaling field or be a mixture of both. Our fits of the eigenvalues $\lambda_{i}$ of the covariance matrix seem to indicate that $C_{q}$ and $\chi_{q}$ are a mixture of a part which scales with $\alpha$, and a part which scales according to $\alpha-1$.

Finally, the present fits of the specific heat at $K_{c}$ yielded a value of $\alpha$ of better accuracy and in better agreement with the hyperscaling value than fits of the specific-heat maxima as used in previous works, ${ }^{10,11}$ which we attribute to our large lattice sizes, the larger number of available data points, and to the fact, that our data and fit was done extremly close to the critical temperature. Moreover, by fitting the energy at $K_{c}$ to eq. (12), we obtained an estimate for $\alpha / \nu$ with a precision so far unpreceeded by direct numerical $\mathrm{MC}$ simulations and in accuracy comparable to hyperscaling predictions.

\section{Acknowledgement}

We are indepted to A.D. Sokal for discussions that initiated this study. W.J. thanks the DFG for a Heisenberg fellowship.

The numerical simulations were performed on the CRAY X-MP and Y-MP of the Konrad-Zuse Zentrum für Informationstechnik Berlin (ZIB), and the CRAY X-MP at the Rechenzentrum der Universität Kiel. We thank all institutions for their generous support. 


\section{References}

${ }^{1}$ B.I. Halperin, in Physics of Defects, Proceedings of the Les Houches Summer Institute (North-Holland, Amsterdam, 1980).

${ }^{2} \mathrm{H}$. Kleinert, Gauge Fields in Condensed Matter (World Scientific, Singapore, 1989).

${ }^{3}$ V.L. Berezinskii, Zh. Eksp. Teor. Fiz. 61, 1144 (1971) [Sov. Phys. - JETP 34, 610 (1972); J.M. Kosterlitz and D.J. Thouless, J. Phys. C6, 1181 (1973); J.M. Kosterlitz, J. Phys. C7, 1046 (1974).

${ }^{4}$ D.R. Nelson and B.I. Halperin, Phys. Rev. B19, 2457 (1979); K.J. Strandburg, Rev. Mod. Phys 60, 161 (1988), and Erratum, ibid. 61, 747 (1989); W. Janke, Int. J. Theor. Phys. 29, 1251 (1990).

${ }^{5}$ M.-h. Lau and C. Dasgupta, J. Phys. A21, L51 (1988); Phys. Rev. B39, 7212 (1989).

${ }^{6}$ S. Ostlund, Phys. Rev. B24, 485 (1981).

${ }^{7}$ A.D. Sokal, private communication.

${ }^{8}$ U. Wolff, Phys. Rev. Lett. 62, 361 (1989).

${ }^{9}$ A.M. Ferrenberg and R.H. Swendsen, Phys. Rev. Lett. 61, 2635 (1988); and Erratum, ibid. 63, 1658 (1989).

${ }^{10}$ P. Peczak, A.M. Ferrenberg, and D.P. Landau, Phys. Rev. B43, 6087 (1991).

${ }^{11}$ C. Holm and W. Janke, Phys. Lett. A173, 8 (1993); preprint FUB-HEP 19/92 / HLRZ 77/92 (Berlin/Jülich 1992), to appear in Phys. Rev. B43, (1993); see also Nucl. Phys. B (Proc. Suppl.) 30, 846 (1993).

${ }^{12}$ B. Berg and M. Lüscher, Nucl. Phys. B190 [FS3], 412 (1981).

${ }^{13}$ K. Chen, A.M. Ferrenberg, and D.P. Landau, UGA preprint 1993.

${ }^{14}$ R.G. Miller, Biometrika 61, 1 (1974); B. Efron, The Jackknife, the Bootstrap and other Resampling Plans (SIAM, Philadelphia, PA, 1982). 
${ }^{15}$ V. Privman, in Finite-Size Scaling and Numerical Simulations of Statistical Systems, edited by V. Privman (World Scientific, Singapore, 1990), p. 4.

${ }^{16}$ W.H. Press, B.P. Flannery, S.A. Teukolsky, and W.T. Vetterling, Numerical recipes - the art of scientific computing (Cambridge Univ. Press, Cambridge, 1986).

${ }^{17}$ J.C. Le Guillou and J. Zinn-Justin, Phys. Rev. B21, 3976 (1980).

${ }^{18}$ For a numerical MC test of the free energy behavior of the three dimensional Ising model see: K.K. Mon, Phys. Rev. Lett. 54, 2671 (1985), and Phys. Rev. B39, 467 (1989).

${ }^{19}$ F.J. Wegner, Phys. Rev. B5, 4529 (1972). 


\section{Tables}

\begin{tabular}{|r|r|r|c|c|c|c|c|c|}
\hline$L$ & \multicolumn{1}{|c|}{$N_{0}$} & \multicolumn{1}{|c|}{$N_{\text {meas }}$} & $\tau_{n}$ & $\langle e\rangle$ & $\langle n\rangle \times 10$ & $\left\langle n_{1}\right\rangle \times 10$ & $\left\langle n_{2}\right\rangle \times 10^{4}$ & $\left\langle n_{3}\right\rangle \times 10^{8}$ \\
\hline 8 & 17 & 50178 & 1.2 & $1.9487(9)$ & $0.9054(18)$ & .88090 & 6.13 & 7.78 \\
12 & 20 & 159575 & 1.6 & $1.9786(3)$ & $0.9845(7)$ & .95689 & 6.90 & 5.80 \\
16 & 40 & 64368 & 1.2 & $1.9905(3)$ & $1.0170(9)$ & .98838 & 7.16 & 7.21 \\
20 & 50 & 27670 & 1.3 & $1.9968(3)$ & $1.0347(6)$ & 1.0053 & 7.35 & 5.42 \\
24 & 50 & 20000 & 1.5 & $1.9998(2)$ & $1.0431(6)$ & 1.0136 & 7.38 & 9.04 \\
32 & 68 & 25403 & 1.5 & $2.0045(2)$ & $1.0561(4)$ & 1.0260 & 7.52 & 6.61 \\
40 & 74 & 21765 & 1.9 & $2.0063(1)$ & $1.0617(3)$ & 1.0314 & 7.59 & 8.04 \\
48 & 93 & 21005 & 1.9 & $2.0074(1)$ & $1.0646(3)$ & 1.0342 & 7.61 & 7.83 \\
56 & 136 & 23795 & 1.6 & $2.0084(1)$ & $1.0674(2)$ & 1.0369 & 7.62 & 6.56 \\
64 & 200 & 26439 & 1.4 & $2.0090(1)$ & $1.0691(1)$ & 1.0385 & 7.64 & 7.18 \\
72 & 150 & 20000 & 1.8 & $2.0093(1)$ & $1.0701(2)$ & 1.0395 & 7.65 & 7.10 \\
80 & 200 & 25431 & 1.7 & $2.00962(4)$ & $1.0709(1)$ & 1.0403 & 7.66 & 6.77 \\
\hline
\end{tabular}

Table 1: Measurement statistics at the simulation point $K_{0}=0.6929: L$ is the linear lattice size, $N_{0}$ is the number of cluster steps between measurements, $N_{\text {meas }}$ stands for the number of measurements, $\tau_{n}$ is the integrated autocorrelation time of the charge density, $\langle e\rangle$ is the energy density, $\left\langle n_{|q|}\right\rangle$ are the observed densities of dual cells with charge $|q|$, and the total defect density $\langle n\rangle$ is defined as $\langle n\rangle=\left\langle n_{1}\right\rangle+4\left\langle n_{2}\right\rangle+9\left\langle n_{3}\right\rangle+\ldots$ 


\begin{tabular}{|r|c|c|c|c|c|}
\hline$L$ & $C$ & $C_{q}$ & $\chi_{q}$ & $\lambda_{1}$ & $\lambda_{2}$ \\
\hline 8 & $2.177(18)$ & $0.712(6)$ & $0.369(3)$ & $2.423(19)$ & $0.1225(8)$ \\
12 & $2.407(12)$ & $0.833(5)$ & $0.430(2)$ & $2.721(14)$ & $0.1261(6)$ \\
16 & $2.562(27)$ & $0.908(10)$ & $0.467(4)$ & $2.900(30)$ & $0.1283(9)$ \\
20 & $2.651(36)$ & $0.951(14)$ & $0.486(6)$ & $3.009(40)$ & $0.1277(7)$ \\
24 & $2.752(40)$ & $0.993(17)$ & $0.506(8)$ & $3.128(47)$ & $0.1297(12)$ \\
32 & $2.832(38)$ & $1.027(14)$ & $0.521(6)$ & $3.223(43)$ & $0.1308(12)$ \\
40 & $2.970(45)$ & $1.081(16)$ & $0.542(7)$ & $3.382(51)$ & $0.1300(9)$ \\
48 & $2.977(44)$ & $1.097(18)$ & $0.553(8)$ & $3.400(51)$ & $0.1304(13)$ \\
56 & $3.142(35)$ & $1.157(12)$ & $0.576(5)$ & $3.587(38)$ & $0.1309(13)$ \\
64 & $3.181(30)$ & $1.173(10)$ & $0.579(5)$ & $3.631(33)$ & $0.1286(10)$ \\
72 & $3.142(55)$ & $1.155(19)$ & $0.574(7)$ & $3.585(62)$ & $0.1315(14)$ \\
80 & $3.182(49)$ & $1.169(16)$ & $0.579(6)$ & $3.630(53)$ & $0.1307(12)$ \\
\hline
\end{tabular}

Table 2: Results for the matrix elements of the covariance matrix $V\left\langle O_{i} ; O_{j}\right\rangle$, with $O_{1}=K e, O_{2}=n$ at $K=0.6930\left(\approx K_{c}\right)$. Also included are the eigenvalues $\lambda_{1}, \lambda_{2}$ of the covariance matrix. 


\section{Figure Headings}

Fig. 1: (a) $C_{q}=T d\langle n\rangle / d T$, and (b) the specific heat $C$ versus $K$ for lattices of size $L=12,40,72$. The values were obtained by reweighting of the runs at $K_{0}=0.6929$.

Fig. 2: (a) $C_{q}$, (b) $C$, and (c) $\chi_{q}$ at $K=0.6930\left(\approx K_{c}\right)$ as function of the lattice size $L$. The solid lines show the best non-linear three-parameter fits to the data.

Fig. 3: (a) $\langle n\rangle$, and (b) $\langle e\rangle$ at $K=0.6930\left(\approx K_{c}\right)$ as function of $L$, together with the best non-linear three-parameter fits to the data.

Fig. 4: (a) $\lambda_{1}$ and (b) $\lambda_{2}$ at $K=0.6930\left(\approx K_{c}\right)$ as function of $L$. Included are also the best non-linear three-parameter fits to the data. 